ESAIM: PROCEEDINGS, October 2007, Vol. 22, 150-154

Gabriel Caloz \& Monique Dauge, Editors

\title{
COMPUTER VISION AND OTOLITH: TOWARD A MODELISATION OF THE MORPHOGENESIS OF ACCRETIONARY PROCESSES
}

\author{
Anatole Chessel $^{1}$, Ronan Fablet ${ }^{1}$ And Frederic CaO $^{2}$
}

\begin{abstract}
Otolith are small stone growing according to an accretionary process located in fishes inner ears from which a lot can be learnt about their biology and ecology. Computer vision is one of the mean of systematic analysis of those stone. This paper describe an approach using partial differential equation and variational methods to reconstruct the morphogenesis of an otolith from an image.

Résumé. Les otolithes sont de petites concrétions calcaires situées dans l'oreille interne des poissons qui peuvent nous en apprendre beaucoup sur leur biologie et leur écologie. La vision par ordinateur est l'une des méthodes d'analyse systématique possible. Nous présentons ici une approche à base d'équation aux dérivées partielles et de méthodes variationnelles permettant de reconstituer la morphogénèse d'un otolithe à partir d'une image.
\end{abstract}

\section{INTRODUCTION}

Otolith are small stones located in fishes inner ears which they use for their spatial localisation. They grow continuously according to an accretionnary process which depend on environmental (e.g. temperature and salinity of the ocean etc) and endogenous (metabolism, sexual maturity etc) conditions and the chemical and optical properties of the deposit at a given time is directly related to those conditions (figure 2, top). Thus the otoliths can play the role of a biological archive from which a lot can be learned about fishes biology and ecology. In particular otolith readings are used daily as a way of estimating the ages of a given fish population, estimation from which stem fish stock evaluation and fisheries resources management.

Several tools are used to decode the archive with respect to environmental and metabolic conditions. Among those, image analysis allow to recover global structural information and is routinely used by human expert to read several hundreds otoliths a year. Computer vision is a field in between artificial intelligence and applied mathematics whose aim is automatic image analysis akin to the one performed by human vision. Otolith image are perfect example on which to apply those methods, as they contain strongly organised visual information using non-local and heterogeneous visual cues.

Thus a long term goal is an automatic analysis of a given otolith image. The framework presented here is a step toward that goal and allow for a global reconstruction of the accretionary process by an estimation of the successive shapes of the otolith. The reconstruction is performed using the tools of constrained regularization driven by the vector field of the tangent to the shape at each point. Classicaly, computer vision rely on contrast information (strong difference of grey- (or color-)level value). But one of the caracteristics of otolith images is to be very weakly contrasted. Thus other kind of information used by human vision have to be relied on to

\footnotetext{
1 IFREMER/LASAA, BP 70, technopole Brest/Iroise, 29280 Plouzane, FR. \{achessel, rfablet\}@ifremer.fr
}

2 IRISA/VISTA, campus de beaulieu, 35042, Rennes, FR. fcao@irisa.fr

(C) EDP Sciences, SMAI 2007 
extract structures. Namely orientation information, which map to each point the tangent of a structure that would pass throught that point.

In a first section we will show how to reconstruct a global orentation field given an otolith image using AMLE (Absolutly Minimizing Lipschitz Extention) interpolation. In the second section we will show how to use that orentation field to drive a variational reconstruction of the otolith morphogenesis.

\section{An Axiomatic of Orientation Interpolation}

Let $I: \Omega \subset \mathbb{R}^{2} \rightarrow \mathbb{R}$ be an image. Let $\theta=\nabla I^{\perp} /\|\nabla I\| \in S^{1}$ (the unit circle of $\mathbb{R}^{2}$ ) be the orientation of the ortogonal to it's gradient. It can be interpreted, where it's well defined, as the tangent to a geometric structure passing throught a point. In a natural image, it would be meaningful mainly on edges, but in otolith images such an orientation can be constructed at each point. It would correspond to the orthogonal of the growth direction or equivalentely to the tangent to the shape the otolith had at that time.

Such a field will be computed in two step. First, we will extract from the image the point where $\theta$ (as defined above) is reliable, using any of the many filters available in the computer vision litterature (a Canny-Deriche filter is used here). Let $D \subset \Omega$ be the set of those extracted points (figure 2, second image). The second step is the extention of the sparse orientation field $\left.\theta\right|_{D}$ to the whole domain $\Omega$.

In [4] an axiomatic approach similar to [3] was developed to find sound interpolation operator by laying out the properties it should follow. Let $\theta: D \rightarrow S^{1}$ be the funtion to be interpolated, let $\phi: S^{1} \rightarrow[0,2 \pi[$ be a parametrisation of $S^{1}$, we are looking for an interpolation operator $E$ extending $\theta$ to $\Omega$ satisfying the following axioms (see [4] for details):

Axiom 1. Comparison principle: $\phi\left(\theta_{1}\right) \geq \phi\left(\theta_{2}\right) \Rightarrow E\left(\Omega, \phi\left(\theta_{1}\right)\right) \geq E\left(\Omega, \phi\left(\theta_{2}\right)\right)$.

Axiom 2. Stability principle: $\Omega^{\prime} \subset \Omega \Rightarrow E\left(\Omega^{\prime}, E(\Omega, \theta) \mid \Omega^{\prime}\right)=E(\Omega, \theta) \mid \Omega^{\prime}$

Axiom 3. Regularity principle: for quadratic function, the infinitesimal action of $E$ is described by a smooth function.

Axiom 4. Translation invariance: $E\left(\Omega-h, \tau_{h}(\theta)\right)=\tau_{h} E(\Omega, \theta)$

Axiom 5. Domain rotation invariance: $E\left(R \Omega, \theta \circ R^{-1}\right)=E(\Omega, \theta) \circ R^{-1}$.

Axiom 6. Zoom invariance: $E\left(\lambda^{-1} \Omega, \lambda \theta\right)=\lambda E(\Omega, \theta)$.

If independance over linear change of parametrisation is supposed, the Absolutely Minimizing Lipschitz Extension (AMLE) is among the possible operators. It is a well studied operator for which existence and unicity results are known ( [3] and reference therein). In particular it satisfies a maximality principle which guarantee that the solution is oscillation free. It is defined as follow.

Let $\Omega \subset \mathbb{R}^{2}$. Let $S^{1}$ be parametrized by angles in $[0,2 \pi[$. Let $D \subset \Omega$ be a set of curves and/or points and $\theta_{0}: D \rightarrow S^{1}$. Then $\theta: \Omega \rightarrow S^{1}$ is the AMLE of $\theta_{0}$ in $\Omega$ if:

$$
\left\{\begin{array}{l}
\nabla^{2} \theta(\nabla \theta, \nabla \theta)=0 \text { in } \Omega \\
\left.\theta\right|_{D}=\theta_{0} \text { on } D
\end{array}\right.
$$

i.e. if the second derivative in the direction of the gradient is null. The mathematical theory only hold if the data $\theta_{0}$ is not surjective, i.e. if it takes value in $S^{1}$ minus one point. If it is not the case, singularities are bound to appear, which the operator, looking for Lipschitz solutions, cannot handle. The AMLE can be seen as a laminar flow orientation operator, not a turbulent flow one. But in practice we are interested in geometric structures which give rise to regular orientation field, thus this necessary condition will be locally fulfilled.

The implementation has been done using a finite difference scheme in a multi-resolution framework (see [4]). Results can bee seen figure 2 third image, visualised through it's flow lines using line integral convolution (LIC, [1]). We can see that the field indeed capture the underlying growth by being at each point tangent to the structure seen in the original image. This field will now be used to reconstruct the growth itself. 


\section{Variational Modelisation of Morphogenesis}

The accretionary growth process is modeled as a potential function $U$ defined over $\mathbb{R}^{2}$ with values in $[O, T]$ such that the level set $\Gamma_{t}(U)$ of $U$ for level $t$ is the shape of the considered biological structure within a given observation plane at time $t$, that is to say

$$
\Gamma_{t}(U)=\left\{p \in \mathbb{R}^{2} \text { such that } U(p)=t\right\}
$$

Intrinsically, such a representation guarantees that the growth is normal to the shape, as commonly assumed for accretionary growth. Note that, in practice, the actual time information might not be available, so that the values of potential function $U$ are not actual time values, but are homogeneous to time.

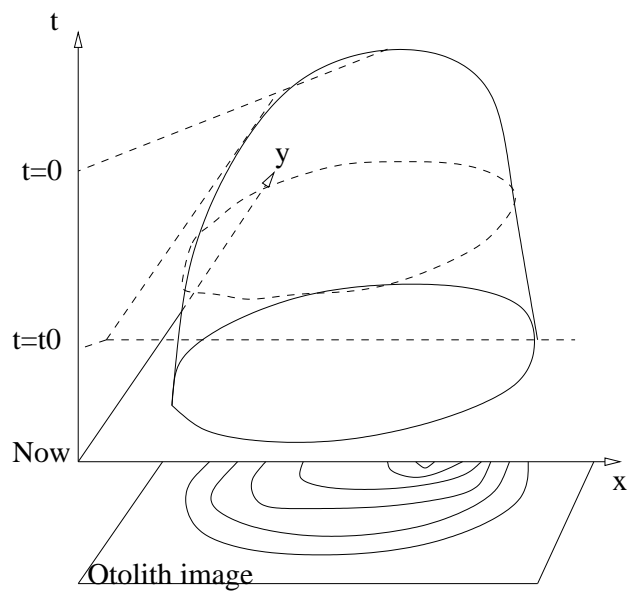

Figure 1

Based on this level-set representation, we aim at estimating the potential function $U$ associated with a given biological structure. Formally, some boundary conditions are assumed to be provided. These conditions include the shapes at times 0 and $\mathrm{T}$, which may be automatically extracted [2]. solving for the reconstruction of $U$ is then stated as the computation of the extension of the boundary values $G_{\mathcal{B}}$ associated with boundary conditions $\mathcal{B}$, to the whole domain $\mathbb{R}^{2}$ according to some energy criterion $E$ :

$$
\widehat{U}=\arg \min _{U \in \mathcal{U}\left(G_{\mathcal{B}}\right)} E(U)
$$

where $\mathcal{U}\left(G_{\mathcal{B}}\right)$ is the set of the functions from $\mathbb{R}^{2}$ to $\mathbb{R}$ which are equal to $G_{\mathcal{B}}$ on boundary set $\mathcal{B}$. Energy $E(U)$ is split into two terms: a regularization term issued from a geometric shape prior and a data-driven term setting orientation-based constraints:

$$
E(U)=E_{R}(U)+\gamma E_{O}(U),
$$

where $\gamma$ is a weighting factor balancing the relative influence of each term. The regularization term is defined as the sum of the perimeter of the growth shapes computed as

$$
E_{R}(U)=\int_{t \in[0, T]} \int_{p \in \Gamma_{t}(U)} 1 .
$$

The second term relies on orientation-based constraints. Let $\theta$ be the orientation field computed in the previous section. As the normal to shape $\Gamma_{t}(U)$ is expected to be orthogonal to orientation $\theta$, the energy term $E_{O}(U)$ is 
computed as follows

$$
E_{O}(U)=\int_{t \in[0, T]} \int_{p \in \Gamma_{t}(U)} \alpha(p) \cdot\left\langle\frac{\nabla U(p)}{|\nabla U(p)|}, \theta(p)\right\rangle^{2}
$$

Minimizing explicitly criterion $E(U)$ is however infeasible in practice, since it would require to extract all the level-lines of $U$. From the co-area formula [5], an equivalent formulation can be derived such that the resulting criterion only involves the spatial derivatives of $U$. More precisely, the minimization of $E(U)$ resorts to:

$$
\widehat{U}=\arg \min _{U \in \mathcal{U}\left(G_{\mathcal{B}}\right)} \int_{p \in \mathbb{R}^{2}}|\nabla U|+\gamma \cdot \alpha \cdot|\nabla U| \cdot\left\langle\frac{\nabla U}{|\nabla U|}, \theta\right\rangle^{2}
$$

This variational setting involves two classical terms: the first one is regularization term set as a the norm of the gradient of the potential function and the second is the data-driven term, which aims at aligning the orientation field of $U$ to orientation constraints $\theta$. In this second term, norm $|\nabla U|$, inherited from the co-area transform, is a weighting factor such that more influence is given to points where the gradient of the level-set representation is high (conversely, where the growth is slow).

Criterion (7) is derived from purely geometric constraints. In addition, level-set representations are contrast invariant (i.e., a contrast change does not affect the geometry of the level-sets but only leads to different indexes for each level-set) [6]. Consequently, additional constraints are required to prevent from retrieving unstable solutions. As constraints on the surface between successive level-lines are equivalent to constraints on first-order statistics $p(U)$ of $U$, minimization (7) will be carried out subject to $p(U)$ is uniform. The choice of a uniform prior is arbitrary. Other priors might be used, for instance priors related to a priori growth laws.

The result can be seen figure 2 bottom. Level-lines (isovalues) of the computed potential are shown on top of the original image. We can see that large pieces of the potential level-lines correspond to structures in the image. Moreover the sequence of the level-lines $\Gamma_{t}, t \in[0, T]$ is an estimation of the changes in the otolith shape during the fish life.

\section{Conclusion}

Computer vision techniques based on partial differential equations and energy minimisation have been show that allow to reconstruct the morphogenesis of an acretionary structure from an image. That reconstruction is based on an orientation field stating the tangent to the wanted structure at each point (or equivalently, if the orthogonal is taken, the direction of growth at a given point). It's based on extracted data from the image extended in $S^{1}$ by the AMLE.

Building a model of morphogenesis is but a first step toward a decoding of the biological archives with respect to environemental and endogenous condition. Next steps include the use of statistical methods to build species-specific model and to have a quantitative assessement of the quality of the sequence of shapes found, and a fusion with data from other analysis methods (chemical, spectroscopic, microscopic etc...) to build an all-in-one model of otolith growth.

\section{REFERENCES}

[1] B. Cabral and L.C. Leedom, "Imaging vector field using line integral convolution," Computer Graphics Proceedings, pp. 263-270, 1993.

[2] F. Cao and R. Fablet. Automatic morphological detection of otolith nucleus. Pattern Recognition Letters, 2006.

[3] V. Caselles, J.M. Morel, and C. Sbert. An axiomatic approach to image interpolation. IEEE Trans. Image Processing, 7(3):376386, 1998.

[4] A. Chessel, F. Cao, and R. Fablet. Interpolation of Orientation : Axiomatic Approach and Applications Journal of Mathematical Imaging and Vision, Submitted, 2006

[5] F. Lin and X. Yang. Geometric Measure Theory: An Introduction. International Press, 2002.

[6] P. Monasse and F. Guichard. Fast computation of a contrast invariant representation. IEEE Trans. on Image Processing, $9(5): 860-872,2000$. 

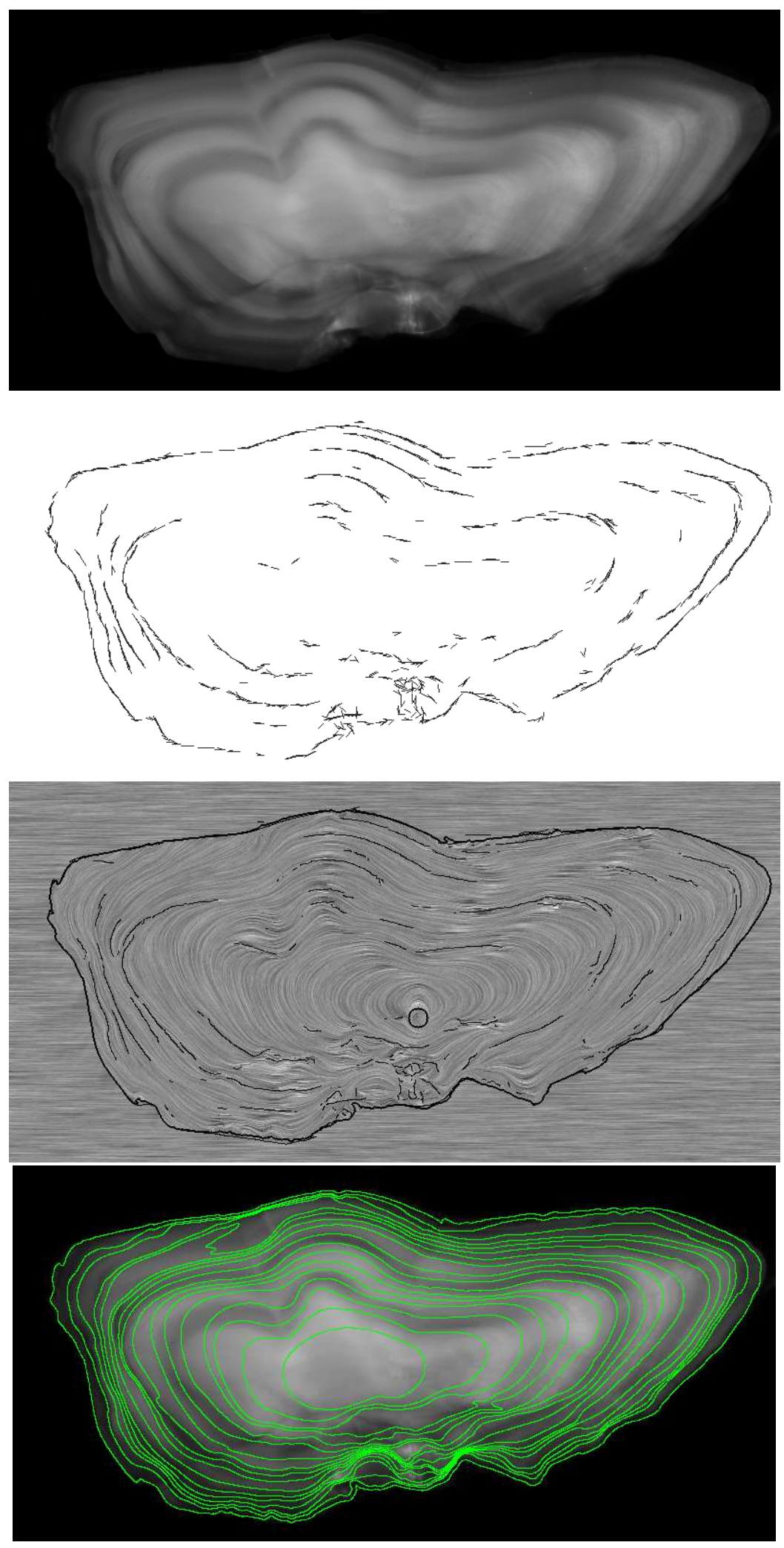

Figure 2. top to bottom: original otolith image, extracted orientation to be extended, field obtained by AMLE, reconstructed shapes on top of original image 